\title{
A ray mapping method for off-axis and non-paraxial freeform illumination lens design.
}

\author{
Karel DesniJder ${ }^{*}$, Peter Hanselaer ${ }^{1}$, and Youri Meuret ${ }^{1}$ \\ ${ }^{1}$ Department of Electrical Engineering (ESAT), Light \& Lighting laboratory, Gebroeders De Smetstraat 1, 9000 Gent, Belgium, \\ *Corresponding author: karel.desnijder@kuleuven.be
}

Compiled December 26, 2018

The ray mapping method for freeform illumination design is an easy and flexible method, but only in the paraxial regime does it results in surface normal fields that are directly integrable into continuous freeform surfaces that provide the desired illuminance distribution. A new mapping scheme is proposed to alter an initial source-target mapping via a symplectic flow of an equi-flux parametric coordinate system. The resulting mapping provides integrable surface normal vector fields for complex off-axis and non-paraxial illumination problems as demonstrated by two freeform lens examples. () 2018 Optical Society of America

http://dx.doi.org/10.1364/ao.XX.XXXXXX

The freeform illumination design problem deals with the question of how to calculate one or more freeform reflective or refractive surfaces in order to redirect the light emitted by a source to produce a certain prescribed illumination. In the past two decades a variety of methods have been described to design freeform illumination components. A recent review paper [1] divides these methods for zero-étendue sources (i.e. point sources or parallel beams) in three separate categories: ray mapping methods, Monge-Ampère equation methods and supporting quadric methods.

The ray mapping method splits the problem in two separate parts: (1) the generation of a ray mapping that connects each incoming ray direction to a specific outgoing ray direction, and (2) the calculation of the freeform surface(s) that realize the corresponding ray redirections. When one knows for each incident ray direction, the corresponding outgoing ray direction, the necessary surface normal can be calculated with Snell's law or the law of reflection. These surface normals can be used to generate the freeform surface via numerical integration [2][3][4]. The ray mapping method is a flexible and easy-to-implement method that can be extended to design freeform lenses in which the raydeflection is divided over multiple freeform surfaces [4][5][6] or freeform lenses with an arbitrary lens contour [7].

The problem with the ray mapping method however is that the resulting normal field $\mathbf{N}$ is typically not directly integrable into a continuous freeform surface if the ray mapping diverges from the paraxial regime [8]. If an optical surface is fitted through such a non-integrable normal field, this results in an illumination distribution that deviates significantly from the desired target distribution. For a normal field to be integrable, it must satisfy the integrability condition [9]:

$$
(\nabla \times \mathbf{N}) \cdot \mathbf{N}=0
$$

Finding a ray mapping that yields an integrable normal field is a hard problem. The Monge-Ampère method avoids integrability problems by directly optimizing the optical surface. This method however requires a set of non-linear partial differential equations to be solved [10,11]. Alternatively, the supporting quadrics $[12,13]$ or cartesian oval [14] method constructs an initial freeform surface as a collection of elliptic or cartesian oval patches from which an integrable mapping can be obtained. For more complex optical systems one needs generalized cartesian ovals that in some cases can be difficult to obtain.

In this letter, a method is put forth that allows to find integrable ray mappings. The method is very general in the sense that it can be applied to any incident wavefront and any prescribed target illumination distribution. The new method is illustrated by designing the required freeform lens for two different non-paraxial illumination cases.

The method starts from an arbitrary known incoming wavefront $W$ which is orthogonal to the incoming ray directions $\mathbf{I}$ as depicted in Fig. 1 If the irradiance of the incoming wavefront is also known, one can calculate the projected irradiance distribution onto a specific source plane orthogonal to the optical axis (z-axis) at a distance $s_{z}$. This projected irradiance distribution is considered as the source distribution $S$. Furthermore the desired target irradiance distribution $T$ onto a specific target plane orthogonal to the optical axis at a distance $t_{z}$ is also known.

The typical starting point of ray mapping methods is an optimal mass transport mapping or curl-free mapping [15] between the source irradiance distribution $S$ and the target irradiance distribution $T$. As described in previous work [7] such a curlfree ray mapping can be split into two separate curl-free mappings: a mapping $\phi(u, v)$ to the source distribution $S$ from a uniform unit square distribution $U(u, v)=1 \forall u, v \in[0,1]$, and a mapping $\psi(u, v)$ to the target distribution $T$ from the same uniform unit square distribution $U$. The mapping $\psi \circ \phi^{-1}$ is then also a curl-free mapping from the source distribution to the target distribution.This is depicted in the left part of Fig. 2. The advantage of this approach is that two unique coordinates $(u, v)$ can be assigned to every ingoing and outgoing light ray. 


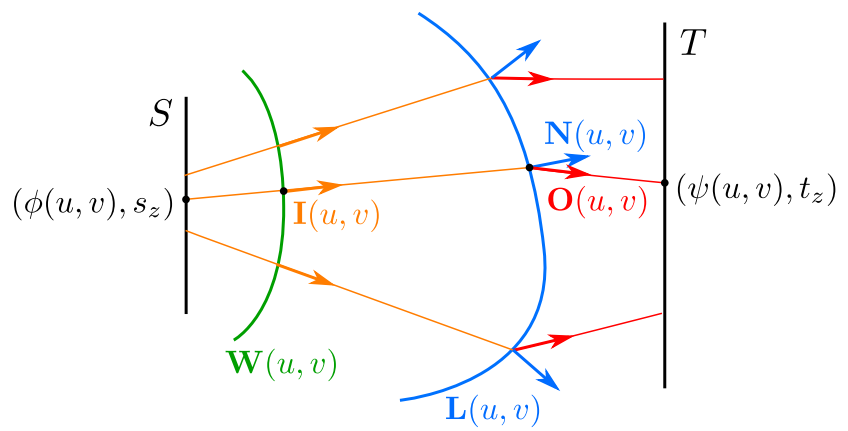

Fig. 1. An ingoing ray $\mathbf{I}(u, v)$ passes through the source plane at the point $\left(\phi(u, v), s_{z}\right)$. At any point along the trajectory of the ray one can construct a wavefront $\mathbf{W}$. If an integrable normal field $\mathbf{N}$ exists, one can construct a lens surface $\mathbf{L}$ such that the ingoing rays $\mathbf{I}(u, v)$ are refracted to outgoing rays $\mathbf{O}(u, v)$ which cross the target plane at the points $\left(\psi(u, v), t_{z}\right)$

The mapping $\psi \circ \phi^{-1}$ can be found as a combination of two optimal mass transport mappings for which different algorithms exist [15] [11]. The algorithm of Wu et al [11] is used.

In order to find an integrable source-target mapping, the initial source-target mapping is altered with an additional symplectic or area-preserving mapping $\mathrm{Y}: U \rightarrow U$ which is schematically depicted on the right side of Fig. 2 Because the mapping $\mathrm{Y}$ is area-preserving, the mapping $\psi \circ \mathrm{Y} \circ \phi^{-1}$ is also a mapping from the source points in $S$ to a new set of target points in $T$ that will leave the resulting distribution $T$ unchanged. The challenge is now to find a symplectic mapping $\mathrm{Y}$ such that the surface normal vector field $\mathbf{N}$ corresponding with $\psi \circ \mathrm{Y} \circ \phi^{-1}$ satisfies Eq. (1).

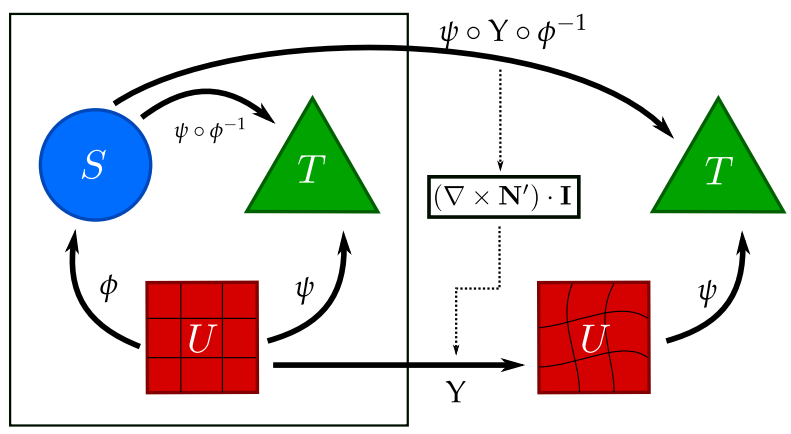

Fig. 2. Schematic representation of the proposed algorithm. The left side (inside the boundary) represents the initial sourcetarget mapping. The right side (outside the boundary) represents the altered source-target mapping that uses the additional symplectic mapping $\mathrm{Y}$.

From the source mapping $\phi(u, v)$ and the source wavefront $\mathbf{W}$ one can derive the ingoing ray directions $\mathbf{I}(u, v)$ at the source plane. From the target mapping $\psi(u, v)$ one can derive the outgoing ray directions $\mathbf{O}=\left(\psi(u, v), t_{z}\right) /\left\|\left(\psi(u, v), t_{z}\right)\right\|$ at the target plane, in case this target plane is at a sufficiently large distance from the freeform surface (i.e. a far-field target distribution is considered). If the target distribution is in the near-field, one can no longer make the assumption that the outgoing rays start in the origin. In this case the starting po- sitions of the outgoing rays are given by the coordinates of the yet unknown lens surface $\mathbf{L}(u, v)$ as $\mathbf{O}=\left(\left(\psi(u, v), t_{z}\right)-\right.$ $\mathbf{L}(u, v)) /\left\|\left(\psi(u, v), t_{z}\right)-\mathbf{L}(u, v)\right\|$. Since the lens surface $\mathbf{L}$ is a priori not known, it has to be obtained iteratively by repeating the algorithm explained in this paper a number of times, starting by using the far-field approximation and using the resulting lens surface in each new iteration. The two examples illustrated in this letter are far-field lenses.

For every pair of ingoing and outgoing ray directions I and $\mathbf{O}$ it is easy to find the required lens surface normal vector $\mathbf{N}$ via Fresnel's law of refraction.

$$
\mathbf{N}=n_{1} \mathbf{I}-n_{2} \mathbf{O}
$$

with $n_{1}$ and $n_{2}$ the refractive indices of the materials before and after the refracting optical surface. As mentioned before, the resulting normal field $\mathbf{N}$ generally does not satisfy the integrability condition. It is therefore necessary to adapt the mapping such that the corresponding $\mathbf{N}$ effectively satisfies Eq. (1).

Since the curl operator is in this case a 3-dimensional operator and the vectors $\mathbf{N}$ are associated with an unknown surface, it is not straightforward to evaluate the curl of N. An expression for the curl of $\mathbf{N}$ that can be easily numerically evaluated is therefore needed.

If Eq. (1) holds, then it is possible to show that

$$
\lambda \mathbf{N} \cdot(\nabla \times \lambda \mathbf{N})=0
$$

also holds, with $\lambda$ an arbitrary scalar function defined over $U$. This means that the normal field $\mathbf{N}$ can be re-scaled locally such that its projection onto $\mathbf{I}$ is everywhere equal to one:

$$
\mathbf{N}^{\prime}=\frac{\mathbf{N}}{\mathbf{N} \cdot \mathbf{I}}
$$

Since the vector projection of the rescaled normal field $\mathbf{N}^{\prime}$ onto I is constant, the curl of $\mathbf{N}^{\prime}$ will be parallel to $\mathbf{I}$ (which is considered to be a unit vector field). This means that if

$$
\left(\nabla \times \mathbf{N}^{\prime}\right) \cdot \mathbf{I}=0
$$

then also $\nabla \times \mathbf{N}^{\prime}=\mathbf{0}$ holds, $\mathbf{N}^{\prime}$ is a conservative vector field and the integrability condition Eq. (1) for $\mathbf{N}^{\prime}$ and $\mathbf{N}$ are both valid.

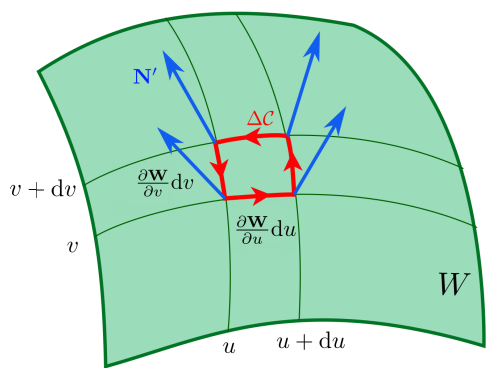

Fig. 3. By integrating the rescaled normal field $\mathbf{N}^{\prime}$ along a contour $\Delta \mathcal{C}$ on the wavefront $\mathbf{W}$ an estimation of the curl of $\mathbf{N}^{\prime}$ can be obtained.

To evaluate Eq. (5) the ingoing wavefront $\mathbf{W}$ orthogonal to the incoming ray directions I is considered (see Fig. 3). By applying the formal definition of the curl of a vector field, the following infinitesimal contour integral is obtained:

$$
\left(\nabla \times \mathbf{N}^{\prime}\right) \cdot \mathbf{I}=\lim _{\Delta S \rightarrow 0} \frac{1}{|\Delta S|} \oint_{\Delta C} \mathbf{N}^{\prime} \cdot \mathrm{d} \mathbf{r}
$$


in which $\Delta S$ is an infinitesimal surface area with $\mathbf{I}$ as normal vector and $\Delta C$ as contour. This contour integral can be written in terms of partial derivatives of $\mathbf{N}^{\prime}$ and $\mathbf{W}$ to $u$ and $v$

$$
\begin{aligned}
\lim _{\Delta S \rightarrow 0} \frac{1}{|\Delta S|} \oint_{\Delta C} \mathbf{N}^{\prime} \cdot \mathrm{d} \mathbf{r} & =\left(\frac{\partial \mathbf{N}^{\prime}}{\partial v} \cdot \frac{\partial \mathbf{W}}{\partial u}-\frac{\partial \mathbf{N}^{\prime}}{\partial u} \cdot \frac{\partial \mathbf{W}}{\partial v}\right) \\
& =C(u, v)
\end{aligned}
$$

as can be understood from Fig. 3. The scalar function $C(u, v)$ is thus proportional to the curl of the normal field $\mathbf{N}^{\prime}$. Since both $\mathbf{N}^{\prime}$ and $\mathbf{W}$ are known vector functions of the parameters $u$ and $v$ the curl can be numerically evaluated.

The ray mapping should thus be altered in such a way that $C(u, v)=0$ for every $(u, v)$ but the resulting target distribution $T$ remains unchanged. As mentioned earlier, this can be done by means of a symplectic transformation $\mathrm{Y}$ of the parametric coordinates $(u, v)$ into a new set of coordinates $\left(u^{\prime}, v^{\prime}\right)$. In this way new outgoing ray directions $\mathbf{O}$ and corresponding surface normals $\mathbf{N}$ will be obtained from the altered source-target mapping $\psi \circ \mathrm{Y} \circ \phi^{-1}$.

A symplectic transformation can be obtained by integration of a Hamiltonian flow. If the domain of $U$ is considered as a phase space with $u$ and $v$ the generalized coordinates, then discrete grid points in $U$ that move according to the Hamiltonian equations of motion will preserve the areas in between them.

Because $\psi$ is a diffeomorphic mapping from the domain $U$ to the target distribution $T$, smooth variations in $(u, v)$ will correspond to smooth variations of the outgoing ray directions $\mathbf{O}(u, v)$ which in turn, via Eq.(2), will result in smooth variations of the normal field $\mathbf{N}$. The equations of motion should transport the outgoing rays and associated target points in such a way that the curl of the rescaled normal field reduces. A direct way to reduce this curl is thus to use the scalar field $-C(u, v)$ as the Hamiltonian and evolve the $(u, v)$ grid points accordingly.

The resulting new coordinates $\left(u^{\prime}, v^{\prime}\right)$ must however remain inside the initial rectangular boundary of $U$. This can be achieved by multiplying $C(u, v)$ with a continuous function $E(u, v)$ that is positive everywhere and falls off continuously to zero on the boundary of the distribution $U$. A simple example of such an envelope function is:

$$
E(u, v)=\sin (u \pi) \sin (v \pi)
$$

This forces the movement of the $\left(u^{\prime}, v^{\prime}\right)$ grid points to be parallel along the border of $U$. Since the border of $U$ is mapped onto the border of $T$ by the initial mapping, the new target points for the border of $U$ will remain on the boundary $\partial T$. The equations of motion that should be considered are thus:

$$
\dot{u}=\frac{\partial(C(u, v) E(u, v))}{\partial v} \quad \dot{v}=-\frac{\partial(C(u, v) E(u, v))}{\partial u}
$$

The two partial derivatives can be numerically calculated for all points $(u, v)$. The equations of motion are applied in an iterative process in which the scalar function $C(u, v)$ is re-evaluated in every time step. Due to the fact that every vector or scalar field is expressed in function of the parameters $(u, v)$ it is difficult to directly integrate Eqs. (10). One way to avoid this difficulty is to consider the coordinates $\left(u^{\prime}, v^{\prime}\right)$ also as functions of $(u, v)$. A simple scheme to update $u^{\prime}$ and $v^{\prime}$ is:

$$
\begin{aligned}
& u^{\prime}(u, v, t+\mathrm{d} t)=u^{\prime}(u+\dot{u} \mathrm{~d} t, v+\dot{v} \mathrm{~d} t, t) \\
& v^{\prime}(u, v, t+\mathrm{d} t)=v^{\prime}(u+\dot{u} \mathrm{~d} t, v+\dot{v} \mathrm{~d} t, t)
\end{aligned}
$$

This indirect way of updating coordinates $\left(u^{\prime}, v^{\prime}\right)$ is area preserving and rotates the mapping locally in the correct direction but requires two extra interpolations every iteration.

As $C(u, v)$ locally gets closer to 0 , so will the movement of the grid-points at those locations. Hence the iterative process will converge to a stationary solution. The final symplectic mapping $\mathrm{Y}$ is thus:

$$
Y(u, v)=\lim _{t \rightarrow \infty}\left(u^{\prime}(u, v, t), v^{\prime}(u, v, t)\right)
$$

This iterative way of reducing the curl of the normal vector field has some resemblance with the optimal mass transfer mapping algorithm that was devised by Haker [15] for 2D distributions .

There are however two difficulties that arise with the symplectic movement. Firstly, the boundary $\partial U$ of the parametric coordinates $(u, v)$ is rectangular and contains corners. The envelope function $\mathrm{E}(\mathrm{u}, \mathrm{v})$ restricts the Hamiltonian flow on the border to be parallel to this border which prohibits movement around the corners. A way to circumvent this issue is to give up the strict condition of symplecticity for these border points. One can then interpolate the border points $\left(u_{b}^{\prime}, v_{b}^{\prime}\right)$ by a $2 \mathrm{D}$ curve $\mathbf{B}(l)=\left(u_{b}^{\prime}(l), v_{b}^{\prime}(l)\right)$ and locally shift these points along the border in the direction that reduces the curl. If the $(u, v)$ grid resolution is high enough, the affected boundary areas are small compared to the overall area. Secondly, for a discrete set of rays, Eqs. (10) must be integrated by means of a symplectic integrator. As $C(u, v) E(u, v)$ is typically not separable, one is limited to implicit methods which can increase the computational cost.

A simple case where the classic ray mapping method fails is the design of a freeform surface that redistributes a collimated beam towards a highly off-axis target distribution. Therefore, a uniform equilateral triangular distribution with a side length of $750 \mathrm{~mm}$ at a distance of $1000 \mathrm{~mm}$ from the freeform lens surface is considered. The center of the triangle is shifted $375 \mathrm{~mm}$ in the $y$-direction which makes this clearly an off-axis target distribution. This configuration, schematically represented in Fig. 4, is clearly a non-paraxial, off-axis illumination problem for which the classic ray mapping method provides inadequate results.

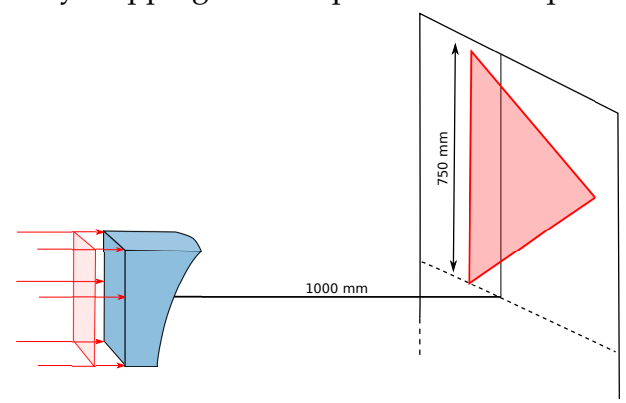

Fig. 4. A uniform, rectangular, collimated beam should be redistributed into a uniform, off-axis, equilateral triangle by a freeform lens surface.

Fig. 5a shows a regular uniform, rectangular grid representing the distribution $U$ with two red lines of constant $u$ and two red lines of constant $v$. Fig. $5 \mathrm{c}$ shows the grid of target points $\psi(u, v)=\left(t_{x}, t_{y}\right)$ obtained via the curl-free mapping $\psi$ from $U$ to $T$. This ray mapping results in a normal vector field that is however not curl-free. Fitting a lens surface to this normal field 
by linear integration results in a lens that produces an irradiance distribution that deviates quite strongly from the targeted uniform triangle as shown in Fig. 5e

On the right side, in Fig. $5 b$, the transformed grid via the symplectic mapping $\mathrm{Y}(u, v)$ is shown. When target points $\left(t_{x}, t_{y}\right)=\psi(\mathrm{Y}(u, v))$ shown in Fig. $5 \mathrm{~d}$ are used to calculate the normal vector field, which is used to construct the lens surface, it clearly results in a much more uniform off-axis triangular distribution shown in Fig. $5 \mathrm{f}$

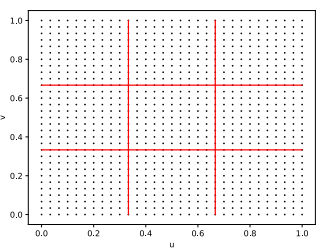

(a) Uniform rectangular grid representing $U$ before symplec-

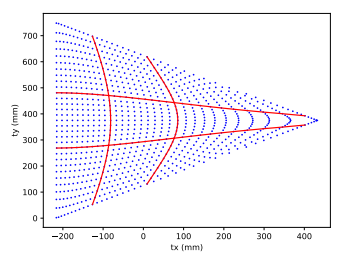

(c) Target points corresponding with the initial mapping $\psi$

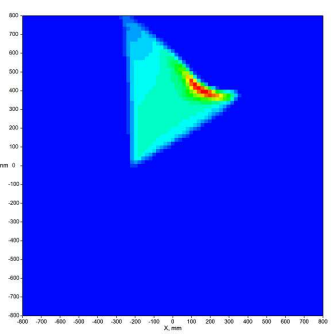

(e) Distribution corresponding with the curl-free mapping $\psi$ tic transformation

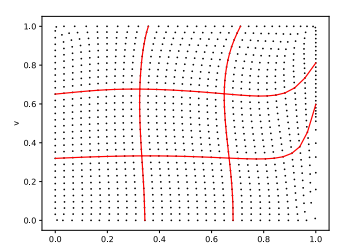

(b) Grid representing $U$ after symplectic transformation $\mathrm{Y}$

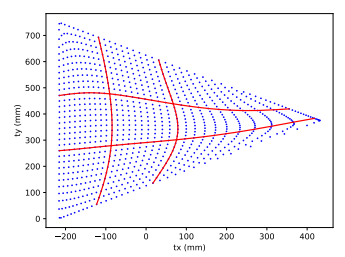

(d) Target points corresponding with the adapted mapping $\psi \circ$ $\mathrm{Y}$

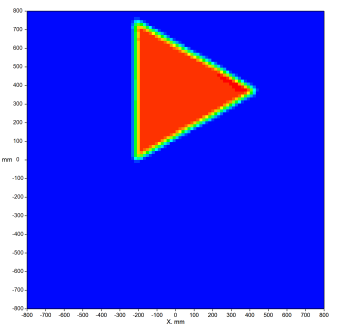

(f) Distribution corresponding with the adapted mapping $\psi \circ$ Y
Fig. 5. a) and b) contain two grids representing the rectangular distribution $U$ before and after the symplectic transformation. c) and d) show the resulting target points. e) and f) show the simulated irradiance distributions in Lighttools 8.6.0

The method can also be used to redirect a source distribution from an arbitrary incoming wavefront into an arbitrary target irradiance distribution. As a second more complex example, a triangular lens is generated that redirects a triangular section of a Lambertian intensity pattern into a distribution that generates a uniform pentagonal pattern with the letters "KUL" on a target plane at a distance of $1000 \mathrm{~mm}$. The pentagon is inscribed in a circle of radius $1000 \mathrm{~mm}$. The triangular source distribution has a maximum acceptance angle of $73^{\circ}$ with the optical axis, meaning that this configuration is clearly also non-paraxial. The triangular beam first refracts on a flat primary surface of the lens before it is refracted by the second freeform surface in the desired irradiance distribution. Even though the light source is a point source, after the first refraction by the flat entrance sur- face, the resulting wavefront is not spherical anymore. If the source plane is chosen to coincide with the primary flat surface, then the wavefront $\mathbf{W}$ after refraction can be represented by:

$$
\mathbf{W}(u, v)=\left(\phi(u, v), s_{z}\right)+\left(\frac{c-n_{2}\left\|\left(\phi(u, v), s_{z}\right)\right\|}{n_{1}}\right) \mathbf{I}_{\mathbf{r}}(u, v)
$$

with $\mathbf{I}_{\mathbf{r}}(u, v)$ the normalized ray directions after refraction. And $c$ a constant optical path length from the source. The lens shape and resulting irradiance distribution can be seen in Fig. 6
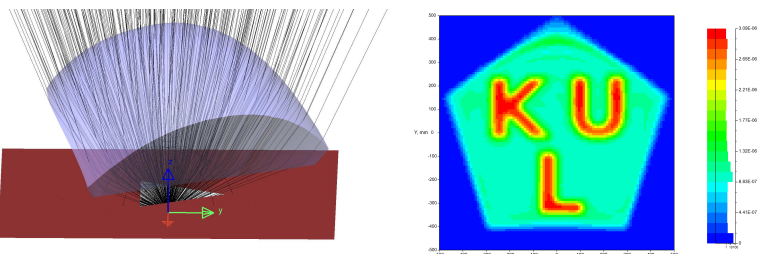

Fig. 6. A plano-convex freeform lens designed with a resolution of $100 \times 100$ points. The point cloud was entered in LightTools, interpolated to a NURBS surface and simulated.

In both cases the freeform surfaces are obtained by a pointby-point second order Euler integration scheme of the normal vector field obtained from the adapted ray mapping, without needing any further optimization. These two examples demonstrate the flexibility of the developed method to create ray mappings that result in integrable normal fields for complex off-axis and non-paraxial freeform illumination problems.

\section{ACKNOWLEDGEMENTS}

This research was supported by VLAIO (Flanders Innovation \& Entrepreneurship) through the O\&O project "Madonna: Methodologies and algorithms for the development and production of next-gens LED armatures (145023)".

\section{REFERENCES}

1. R. Wu, Z. Feng, Z. Zheng, R. Liang, P. Benítez, J. C. Miñano, and F. Duerr, Laser \& Photonics Rev. 12, 1700310 (2018).

2. C. Bösel and H. Gross, Opt. Express 24, 14271 (2016).

3. Z. Feng, L. Huang, M. Gong, and G. Jin, Opt. Express 21, 14728 (2013).

4. Z. Feng, L. Huang, G. Jin, and M. Gong, Opt. Express 21, 28693 (2013).

5. A. Bäuerle, A. Bruneton, R. Wester, J. Stollenwerk, and P. Loosen, Opt. Express 20, 14477 (2012).

6. A. Bruneton, A. Bäuerle, R. Wester, J. Stollenwerk, and P. Loosen, Opt. Express 21, 10563 (2013).

7. K. Desnijder, P. Hanselaer, and Y. Meuret, Opt. Lett. 42, 5238 (2017).

8. A. Bruneton, A. Bäuerle, R. Wester, J. Stollenwerk, and P. Loosen, Opt. Lett. 38, 1945 (2013).

9. H. Ries and J. Muschaweck, J. Opt. Soc. Am. A 19, 590 (2002).

10. R. Wu, L. Xu, P. Liu, Y. Zhang, Z. Zheng, H. Li, and X. Liu, Opt. Lett. 38, 229 (2013).

11. R. Wu, Y. Zhang, M. M. Sulman, Z. Zheng, P. Benítez, and J. C. M. nano, Opt. Express 22, 16161 (2014).

12. V. I. Oliker, Trends Nonlinear Analysis, M. Kirkilionis, S. Kromker, R Rannacher, F. Tomi, eds. p. 193-222 (2003).

13. F. R. Fournier, W. J. Cassarly, and J. P. Rolland, Opt. Express 18, 5295 (2010).

14. D. Michaelis, P. Schreiber, and A. Bräuer, Opt. Lett. 36, 918 (2011).

15. S. Haker, L. Zhu, A. Tannenbaum, and S. Angenent, Int. J. Comput. Vis. 60, 225 (2004). 


\section{FULL REFERENCES}

1. Y. Zhang, S. Qiao, L. Sun, Q. W. Shi, W. Huang, L. Li, and Z. Yang, "Photoinduced active terahertz metamaterials with nanostructured vanadium dioxide film deposited by sol-gel method," Opt. Express 22, 11070-11078 (2014)

2. Optical Society, "OSA Publishing," http://www.osapublishing.org.

3. P. Forster, V. Ramaswamy, P. Artaxo, T. Bernsten, R. Betts, D. Fahey, J. Haywood, J. Lean, D. Lowe, G. Myhre, J. Nganga, R. Prinn, G. Raga, M. Schulz, and R. V. Dorland, "Changes in atmospheric consituents and in radiative forcing," in Climate Change 2007: The Physical Science Basis. Contribution of Working Group 1 to the Fourth assesment report of Intergovernmental Panel on Climate Change, S. Solomon, D. Qin, M. Manning, Z. Chen, M. Marquis, K. B. Averyt, M. Tignor, and H. L. Miler, eds. (Cambridge University Press, 2007).

4. R. McKay, "X-ray crystallography," Ph.D. thesis, Princeton University (1982).

5. V. S. C. Manga Rao and S. Hughes, "Single quantum-dot Purcell factor and $\beta$ factor in a photonic crystal waveguide," Phys. Rev. B 75 (2007). 